\title{
Does magnetic resonance imaging increase core body temperature in children? Results of the administration of propofol and ketofol: a randomized clinical study
}

\author{
Çiğdem Akyol Beyoğlu' ${ }^{1 \oplus}$, Pınar Kendigelen ${ }^{1 \oplus}$, İlhan Beyoğlu ${ }^{1 \oplus}$, Fatiş Altındaş ${ }^{1 \oplus}$, \\ Sebuh Kuruoğlu' ${ }^{2 \oplus}$, Güner Kaya ${ }^{1 \oplus}$ \\ Departments of ${ }^{1}$ Anesthesiology and Reanimation and ${ }^{2}$ Radiodignostic and Interventional Radiology Istanbul University Cerrahpasa \\ Faculty of Medicine, Istanbul, Turkey.
}

\begin{abstract}
Background and objectives. Magnetic resonance imaging (MRI) may cause a temperature increase in the imaging area, while intravenous anesthetics may develop a tendency for hypothermia, especially in the pediatric population. The effect of different anesthetics on core body temperature in children during these procedures remains controversial. We examined the effect of propofol and ketofol on core body temperatures in a pediatric population during MRI. Our hypothesis was that the increase in body temperature will be more prominent in pediatric patients receiving ketofol than in those receiving propofol.

Methods. This was a randomized, prospective, double-blind study in pediatric patients aged 6 months to 10 years. The patients were American Society of Anesthesiologist (ASA) physical class I-II who had undergone MRI under anesthesia at the Cerrahpasa School of Medicine, MRI Area, between August 2014 and February 2016. Patients were assigned to one of two groups: Group I (propofol group) and Group II (ketofol group). MRIs were performed with a 1.5 Tesla $(\mathrm{T})$ device. Bilateral tympanic membrane temperature measurements before and after the procedure were performed.
\end{abstract}

Results. Body temperature decreased in both groups after MRI. Clinically significant hypothermia or hyperthermia was not observed in any of the patients.

Conclusion. Temperature monitoring is not necessary for every patient being imaged. However, temperature changes should be closely monitored in high-risk patients.

Key words: magnetic resonance imaging, body temperature, ketofol, propofol, sedation.

Magnetic resonance imaging (MRI) is a commonly used imaging modality for diagnosis in pediatric patients. The imaging area must remain stationary to improve the image quality. Therefore, MRIs are frequently performed on children under general anesthesia or sedoanalgesia. ${ }^{1}$ During imaging, the body absorbs radiofrequency (RF) waves emitted from the MRI device, resulting in a temperature increase in the imaging area. This

Çiğdem Akyol Beyoğlu

akyolbeyoglu@gmail.com

Received 15th September 2018, revised 9th March 2019, 5th April 2019, 18th May 2019, accepted 20th May 2019. increase can cause a significant rise in body temperature since the ratio of body surface area (BSA) to weight is high in children. ${ }^{1}$ However, body temperature can also drop because the environmental temperature during the MRI should be low $\left(20^{\circ} \mathrm{C}\right)$ for the device to work effectively. Active heating devices are not used since they are incompatible with the MRI device, and intravenous (IV) anesthetics negatively affect thermoregulation. ${ }^{1}$

Sedation with IV anesthetics disrupts thermoregulation. ${ }^{2,3}$ Most IV anesthetics cause peripheral vasodilatation, resulting in a redistribution of body temperature from central to peripheral body compartments, thereby 
leading to hypothermia..$^{4-6}$ Thermodysregulation is frequently observed after the induction of anesthesia with IV propofol, which has a prominent peripheral vasodilation effect. ${ }^{7-10}$

Ketamine is different from other IV anesthetics and does not impair thermoregulation because it stimulates the sympathetic nervous system and increases peripheral vascular resistance. ${ }^{7,8}$ It has been shown that the use of ketamine in combination with propofol for maintenance of anesthesia inhibits the development of hypothermia., ${ }^{71}$

Our hypothesis was that the increase in body temperature that occurs during MRI will be more prominent in pediatric patients receiving ketofol than in those receiving propofol and, in turn, the risk of hypothermia will be lower in these patients. Our first aim was to evaluate the effects of a ketamine and propofol mixture (ketofol) on body temperature in pediatric patients undergoing imaging with a 1.5 Tesla $(\mathrm{T})$ MRI device. Our second aim was to determine factors, such as age, gender, BSA, imaging area, and shot duration, that may affect body temperature.

Sedative drugs and general anesthetics applied to pediatric patients for immobilization and absorption of RF waves emitted from the MRI device have varying effects on body temperature. By comparing the effects of ketamine and propofol, we think our study can bring new insight to the current literature concerning the confusing effects general anesthetics have on body temperature during MRIs.

Ketamine is different from other general anesthetics as it does not affect body temperature. Unfortunately, to date, there is very little information in the literature concerning the effects of ketamine on body temperature during MRI.

\section{Material and Methods}

The study was approved by Cerrahpasa Medical Faculty Ethics Committee on 1 July
2014 (83045809/604.01/01/118099) with written informed consent from parents. The study was registered clinicaltrials.gov with registration number NCT02931786. Then, the study was conducted prospectively, randomly, doubleblindly in pediatric patients aged 6 months to 10 years with ASA physical class I-II who underwent MRI under anesthesia. Allergy to IV anesthetics, severe cardiac or pulmonary disease, high intracranial pressure or epilepsy, initial body temperature of $37.5^{\circ} \mathrm{C}$ and above were exclusion criteria.

Parents accompany children in the sedation area and anesthesia induction is started with inhalation anesthesia in the meantime parents move to the waiting hall. Sevoflurane inhalation anesthesia was performed after the patients were monitored in the sedation area (HR, SPO2, NIBP). Hemodynamic monitoring continued during MR imaging. All patients underwent iv cannulation together with sevoflurane inhalation induction before entering the MRI unit. $0.1 \mathrm{mg}^{-\mathrm{kg}}$ midazolam (Zolamid, Defarma, Turkey) and $0.01 \mathrm{mg}^{-\mathrm{kg}}$ atropine (Atropine Sulfate, Biofarma, Turkey) were administered intravenously. Patients were divided into two groups according to the sealed envelope method: Group I (propofol group) and Group II (ketofol group). Propofol $1 \mathrm{mg}^{-\mathrm{kg}}$ (Propofol $1 \%$, Fresenius, Germany) was administered intravenously to Group I, and $0.1 \mathrm{ml}^{-\mathrm{kg}}$ of a mixture (ketofol) of propofol and ketamine (Ketalar 500 mg/10 ml, Pfizer, USA) was administered intravenously to Group II.

There is $5 \mathrm{mg}$ propofol and $5 \mathrm{mg}$ ketamine in 1 $\mathrm{ml}$ of ketofol solution we used in the study.

The sedation level of the patients was evaluated according to the Children's Hospital of Wisconsin Sedation Scale ${ }^{12}$ (Table I), and the patients with a sedation score of 3 and below were taken to the MRI unit. Evaluation of sedation level and record of hemodynamic parameters were done by the same anesthesiologist blinded to the study groups. Magnetic resonance imaging was performed with the Siemens MAGNETOM Avanto 1.5T (Siemens Healthcare 
Table I. Children's Hospital of Wisconsin Sedation Scale.

6 Spontaneous agitated, anxious, in pain without stimulus

5 Spontaneous awake and calm without stimulus

4 Drowsy with eyes open or closed, easily aroused with mild to moderate verbal stimulus

3 Drowsy, arousable with moderate tactile or loud verbal

2 Can be aroused to consciousness but slow with sustained painful stimulus

1 Can be aroused but not to consciousness with sustained painful stimulus

0 Unresponsive to painful stimuli

Sector, Henkestraße, Erlangen, Germany). For the patients with a sedation score of 4 and above, $0.5 \mathrm{mg}^{-\mathrm{kg}}$ propofol was administered intravenously to Group I, and $0.05 \mathrm{ml}^{-\mathrm{kg}}$ of a mixture (ketofol) of $5 \mathrm{mg} / \mathrm{ml}$ propofol and $5 \mathrm{mg} / \mathrm{ml}$ ketamine was administered intravenously to Group II. Bilateral tympanic membrane temperature measurements (Genius TM 2, Covidien MN, USA) before entering the MRI unit were performed and recorded by an anesthesiologist blinded to study groups. Patients were taken to the magnetic room with the same type of single-layer cotton clothes, and headphones were plugged to cover both ears. No patients were actively warmed, and all the patients were covered with a hospital blanket up to their shoulders. The temperature of the magnetic room was kept at $20^{\circ} \mathrm{C}$, and the humidity level was $<50 \%$. $4 \mathrm{lt} / \mathrm{min}$ oxygen was given to the patients with the appropriate type of facemask. Heart rate and peripheral oxygen saturation (SpO2) were monitored during MRI. Noninvasive blood pressure values at the start and end of the procedure were recorded. Also, the additional warning was avoided during MRI.

After MRI was completed, the patients were removed from the magnetic room and bilateral tympanic membrane temperature measurements were repeated. During temperature measurement, the child's auricle was slightly pulled backward and upward by holding its superior part, and thermometer sensor was slightly pushed into the ear. A few seconds after pressing the button which initiates the measurement, it was removed from the ear and the measured value was recorded. Temperature measurements before and after imaging and patient follow-up were performed by the same anesthesiologist. When there was a difference of $1{ }^{\circ} \mathrm{C}$ between temperature measurements, the measurement was repeated and the two values were averaged. After the measurement was completed, the sedation score was re-evaluated and re-recorded.

Age, gender, body surface area, imaging area and shot duration of the patients were recorded.

\section{Statistical Data Analysis}

Data obtained in the study were analyzed using SPSS (Statistical Package for Social Sciences) for Windows 22.0 program. The number, percentage, mean, standard deviation was used as descriptive statistical methods in the evaluation of the data.

The t-test was used to compare continuous (or quantitative) data between two independent groups. The difference between repeated measures was analyzed by the paired t-test.

The obtained findings were evaluated within a $95 \%$ confidence interval and at a significance level of $\mathrm{p}<0.05$.

Sample size estimation:

On the basis of a prior study ${ }^{1}$ we considered a difference of $0.5^{\circ} \mathrm{C}$ between the two groups after general anesthesia in MRI unit. We decided to include at least 27 patients for each group in the study with a confidence interval of $95 \%$ identifying at a two-tailed alpha level of 0.05 ( $G^{*}$ Power 3.1.9.4). Therefore, we planned to study a minimum of 60 patients predicting the loss of follow up. 


\section{Results}

Our study was performed in 90 patients undergoing MRI in the MRI unit of our hospital for various reasons between June 2014 and February 2016. During this time, 5 children with intracranial mass, 6 children with epilepsy, and 4 children with a body temperature of $37.5^{\circ} \mathrm{C}$ before imaging were excluded from the study. Seventy-five children who fulfilled the inclusion criteria were evaluated in the final analysis (Fig. $1)$.

There were no significant differences between groups' demographic data in terms of gender distribution, age and body surface area $(p=0.01$, $\mathrm{p}=0.06, \mathrm{p}=0.06$ ) (Table II).
There was no significant difference between groups in means of peripheral oxygen saturations and heart rates in any time intervals $(\mathrm{p}>0.5, \mathrm{p}>0.5)$.

There was no significant difference in sedation scores before and after MRI according to groups $(\mathrm{p}=0.12, \mathrm{p}=0.55)$.

While systolic blood pressure (SBP) before MRI was significantly higher in Group II than in Group I, there was no significant difference between the groups in terms of SBP after MRI $(\mathrm{p}=0.001, \mathrm{p}=0.809)$.

While diastolic blood pressure (DBP) before MRI was significantly higher in Group II than

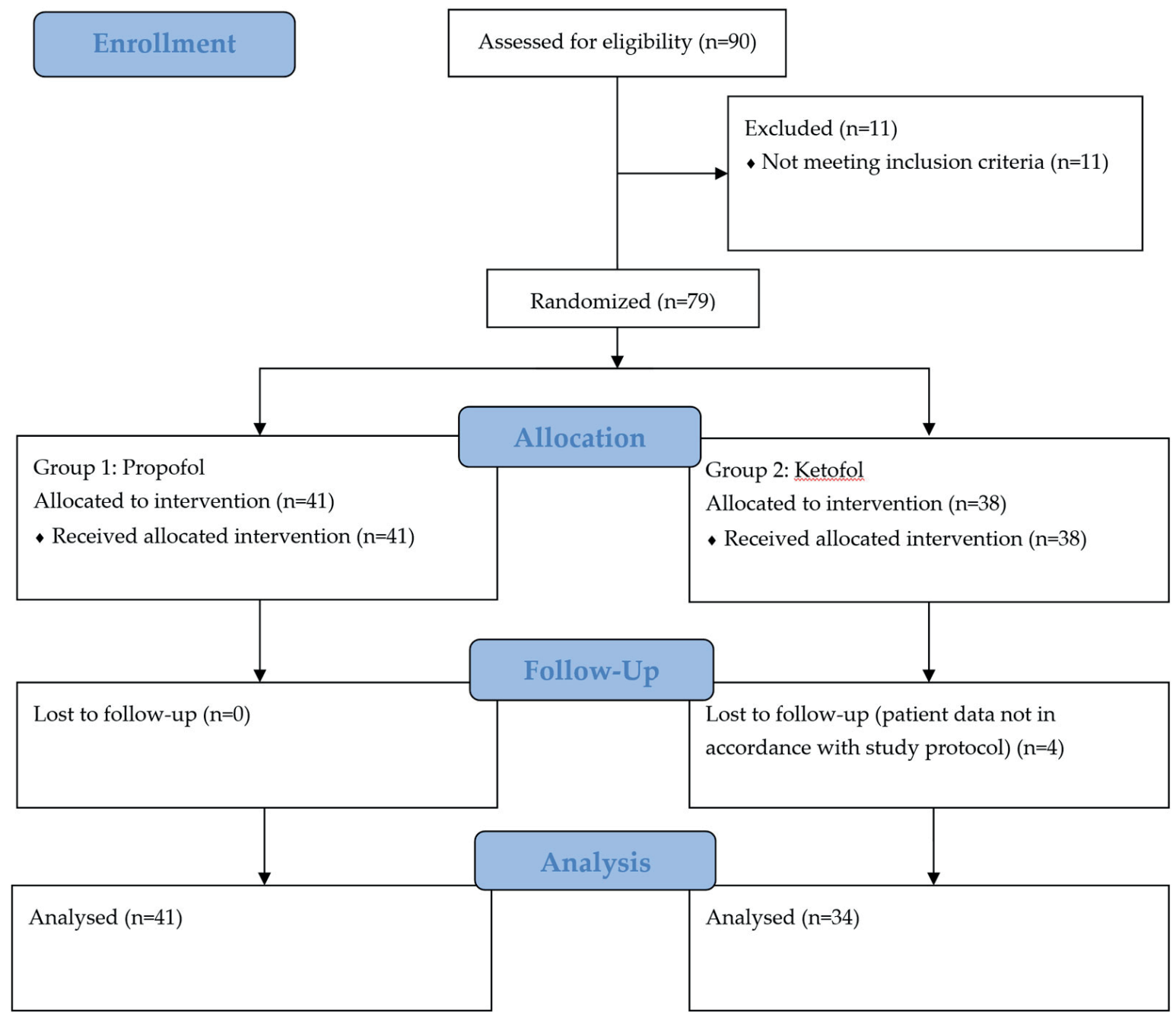

Fig. 1. Flow diagram of the study. 
Table II. Demographic data.

\begin{tabular}{lccc}
\hline & Group I (n: 41) (propofol) & Group II (n: 34) (ketofol) & $\mathrm{p}$ \\
\hline Gender $(\mathrm{M} / \mathrm{F})^{*}$ & $19 / 22$ & $21 / 13$ & 0.01 \\
Age $($ month) & $38.76 \pm 19.47$ & $39.94 \pm 21.24$ & 0.06 \\
Body Surface Area $\left(\mathrm{kg} / \mathrm{m}^{2}\right)$ & $0.57 \pm 0.11$ & $0.62 \pm 0.12$ & 0.06 \\
\hline
\end{tabular}

${ }^{*}$ M: Male, F: Female 15

in Group I, there was no significant difference between the groups in terms of DBP after MRI $(p=0.001, p=0.068)$.

While 30 patients underwent MR imaging of the brain and 11 patients underwent MR imaging of the other regions (spine, abdomen, pelvis) in Group I, 14 patients underwent MR imaging of the brain and 20 patients underwent MR imaging of the other regions (spine, abdomen, pelvis) in Group II. The mean shot duration was $21.15 \pm 8.7$ minutes in Group I and

$20.24 \pm 6.3$ minutes in Group II, respectively. There was no significant difference between the groups in terms of mean shot duration $(p=0.7)$.

In Group I, the mean right tympanic membrane temperatures before MRI were significantly higher than the mean right tympanic membrane temperatures after MRI ( $\mathrm{p}<0.0001)$. In Group II, the mean right tympanic membrane temperatures before MRI were significantly higher than the mean right tympanic temperatures after MRI ( $p<0.022)$. We found a statistically significant difference between the temperatures of two groups after MRI

$(p=0.001)$. The temperature decrease was more prominent in Group I compared to Group II (Table III).

In Group I; mean of SBP was $88.4 \pm 10.2$ before MRI and was $84.5 \pm 9.9$ after MRI. In Group II; mean of SBP was $96.4 \pm 6.7$ before MRI and was $85.2 \pm 14.4$ after MRI.
In Group I; mean of DBP was $49.8 \pm 8.5$ before MRI and was $48.8 \pm 9.1$ after MRI. In Group II; mean of DBP was $58.2 \pm 13$ before MRI and was $52.1 \pm 6.9$ after MRI.

Propofol or ketofol was additionally given to 3 patients with a sedation score of 4 and above in Group I and to 4 patients with a sedation score of 4 and above in Group II. All the patients were asked to remain completely inactive to obtain high-quality images. None of the patients developed any respiratory or cardiac complications.

\section{Discussion}

When 75 pediatric patients included in our study were examined, the tympanic membrane temperatures were significantly decreased after MRI in both groups. However, clinically significant hypothermia or hyperthermia was not observed in any of the patients.

The incidence of hypothermia in children undergoing MRI has not been clearly established to date and thus, remains a subject of debate. Studies on this subject have been mostly performed with oral or rectal sedatives. However, some studies have reported a decrease in body temperature of $21-52 \%$ in children under anesthesia during MRI., ${ }^{9,13}$ Hypothermia should be avoided, especially in premature infants. Preterm infants are more sensitive to the negative effects of cold stress

Table III. The mean right tympanic membrane temperatures before and after MRI according to groups.

\begin{tabular}{lccc}
\hline Right tympanic membrane temperature $\left({ }^{\circ} \mathrm{C}\right)$ & Group I & Group II & $\mathrm{p}$ \\
\hline Before MRI & $36.08 \pm 0.45$ & $36.24 \pm 0.36$ & 0.09 \\
After MRI & $35.67 \pm 0.46$ & $36.11 \pm 0.44$ & 0.001 \\
$\mathrm{p}$ & 0.0001 & 0.022 & \\
\hline
\end{tabular}


due to having thinner skin and limited fat stores. $^{15}$

The results of our study are similar to a study done by Acar et al. ${ }^{13}$. In both studies, body temperatures decreased under MRI, but the decreases were not clinically important.

The results we obtained in our study are not compatible with some previous studies that investigated the effect of MRI on body temperature. ${ }^{1,16-18}$ Machata et al. ${ }^{1}$ evaluated children aged between 1 month and 6 years, and reported increases in core body temperature with MRI. The reason behind this increase may have been due to the younger age of the patients. Another case by Kussman et al. ${ }^{18}$ examined a child scanned for 95 minutes for a cardiac MRI. In this case, it seems that the long scanning duration was responsible for hyperthermia in this patient. Another explanation for this difference may be due to the fact that the magnetic field strength of the MRI device used in some studies is $3 \mathrm{~T} .{ }^{1,9}$ The MRI device emits RF radiation (RFR) to the body region to be imaged in a strong magnetic field and works on the principle that the tissues absorb these RF waves and return the energy they receive. In a $3 \mathrm{~T}$ MRI device with a high magnetic field strength, the imaging area is more rapidly exposed to a stronger magnetic field and thus, the absorption of RF waves by the body and the increase in temperature are more intense in $3 \mathrm{~T}$ MRI devices than in $1.5 \mathrm{~T}$ MRI devices. ${ }^{1}$ In our study, the tympanic membrane temperatures did not increase, possibly because the imaging was performed by an MRI device with a magnetic field strength of $1.5 \mathrm{~T}$.

In a previous study investigating the effects of MRIs performed under anesthesia on body temperature in pediatric patients, there was a body temperature decrease after imaging. ${ }^{14}$ Although this is consistent with the results of our study, $1.5 \mathrm{~T}$ and $3 \mathrm{~T}$ MRI devices were used together in the previous study, and their effects on body temperature were not compared between the $1.5 \mathrm{~T}$ and $3 \mathrm{~T}$ MRI devices. In our study we used a $1.5 \mathrm{~T}$ MRI device on all patients, a fact we believe is important in terms of standardization of the study.

To ensure complete inactivity in the MRI units, IV anesthetics, especially propofol, are frequently used..$^{19}$ In some studies where IV anesthetics were not used for sedation, it was reported that body temperatures increased during MRI. ${ }^{17,18}$ The thermoregulatory center is depressed with the induction of anesthesia. Moreover, peripheral vasodilatation occurs with other IV anesthetics, except for ketamine, resulting in a redistribution of body temperature from central to peripheral body compartments. ${ }^{7,8}$ We found a statistically significant difference between temperature changes of the two groups after MRI. This result suggests that propofol is more effective in producing a body temperature decrease during MRI than ketofol. This finding is in line with the previous literature. ${ }^{21,22}$

The expected effect after the introduction of anesthesia is a decrease in body temperature that is dependent on the drug used and its dose. $^{7,8}$ In the current study, we evaluated the level of anesthesia depth according to the Children's Hospital of Wisconsin Sedation Scale and sent only children under deep sedation to the imaging room. We think that since body temperatures decreased in most of the patients in our study, this decrease may be related to the sedation level. The level of anesthesia depth was not recorded in previous studies that showed increases in body temperature after MRIs. ${ }^{15-17}$ Young children under deep sedation are more susceptible to hypothermia and thus, body temperature should be monitored in such patients. ${ }^{22}$ There is no risk of hypothermia in children undergoing moderate sedation. ${ }^{22}$

The studies in which chloral hydrate and inhalation anesthetics were used for sedation under MRI have typically concluded that MRI increases core body temperature. ${ }^{14,17}$ We feel this result, which is incompatible with our study, may be due to the use of chloral hydrate and inhalation anesthetics instead of IV anesthetics for sedation in the past two studies. ${ }^{14,17}$ All general anesthetics impair autonomic thermoregulatory 
control. ${ }^{24}$ However, most studies investigating the effects of inhalation anesthetics on thermoregulation have been performed with halothane, desflurane, and isoflurane. ${ }^{24-30}$ In addition, Ozaki et al. ${ }^{31}$ showed that age had more of an effect on thermoregulation compared to sevorane/nitrous oxide anesthesia. It has also been reported that isoflurane is more likely to impair thermoregulation compared to sevoflurane. ${ }^{32}$ Furthermore, volatile anesthetics do not affect the peripheral shunt flow, which plays a role in heat regulation. ${ }^{26-33}$

Lo et al. ${ }^{14}$ did not report how long and under which inhalation anesthetic they used in their study, and they concluded that the decrease in body temperature was associated with a younger age and lower temperature before the MRI. The other patients in their study had an increase in body temperature. ${ }^{14}$ Moreover, the mean age of patients was lower in both studies compared to our study. ${ }^{14,17}$ The increase in BSAto-weight ratio, which is inversely proportional to age, may increase the absorption of RF energy from the MRI device. ${ }^{1,17,18}$ The IV anesthetics we used in our study may mask the temperature increase resulting from this energy absorption. However, there were only three infants in Group I and four in Group II in our study. Therefore, we think that the higher mean age of the children in our study may have caused a lower RF energy absorption compared to other studies. ${ }^{14,17}$

All IV anesthetics, except for ketamine, disrupt thermoregulation. ${ }^{711}$ In some studies, the effects of ketamine and other IV anesthetics on the thermoregulatory center were found to be similar. ${ }^{34,35}$ In our study, body temperature did not increase in the ketofol group, which may have been due to the low strength of the $1.5 \mathrm{~T}$ MRI device. However, another explanation may be that we used a single bolus dose of ketamine and not an infusion. Therefore, we were able to prevent children from having significant hyperthermia in both groups.
In this study, the average age of the children was 38 and 39 months in the ketofol and propofol groups, respectively. The older age may have caused a decreased absorption of RF energy compared to younger children due to the BSAto-weight ratio, resulting in a more pronounced drop in body temperature under anesthesia. ${ }^{1}$ The shot duration was short and similar in both groups. This may be the reason for the lack of profound decreases in temperature in both groups. Blankets were enough to keep body temperatures within safe limits in children undergoing MRI in our study.

One of the limitations of our study is that we did not measure the temperature in body regions other than the head and neck in cases where the cranial region was imaged. The necessity of using devices compatible with the MRI device in imaging units increases the cost and limits the use of certain devices, such as rectal thermometers, that measure temperatures from various body regions. Another limitation of our study is that the strength of the MRI device was $1.5 \mathrm{~T}$. Previous studies on this subject have shown that a $3 \mathrm{~T}$ MRI device has a more pronounced effect on body temperature. . $^{1,9,18}$

In our study, the effects of propofol and ketofol on temperature changes during MRI were not clinically significant. Although factors such as the characteristics of the MRI device, the age of the patient, and the imaging area are more effective on temperature change, this issue needs to be further evaluated. Temperature monitoring may not be necessary for every patient being imaged; however, temperature changes should be closely monitored, especially in high risk patients. Cold stress causes an increase in oxygen consumption and metabolic acidosis. Therefore, hypothermia brings a potential risk for newborns and infants, especially if they are preterm, due to their thinner skin and limited fat stores. Hyperthermia is also deleterious for brain injury patients. ${ }^{22}$ We suggest temperature monitoring of newborns, preterm infants, and brain injury patients during MRI. 


\section{REFERENCES}

1. Machata AM, Willschke H, Kabon B, Prayer D, Marhofer P. Effect of brain magnetic resonance imaging on body core temperature in sedated infants and children. Br J Anaesth 2009; 102: 385-389.

2. Bissonnette B, Sessler DI. Thermoregulatory thresholds for vasoconstriction in pediatric patients anesthetized with halothane or halothane and caudal bupivacaine. Anesthesiology 1992; 76: 387-389.

3. Sessler DI. Perioperative heat balance. Anesthesiology 2000; 92: 578-596.

4. Matsukawa T, Sessler DI, Sessler AM, et al. Heat flow and distribution of during induction of general anesthesia. Anesthesiology 1995; 82: 662-673.

5. Vassilieff N, Rosencher N, Sessler DI, Conseiller C, Lienhart A. Nifedipine and intraoperative core body temperature in humans. Anesthesiology 1994; 80: 123-128.

6. Kurz A, Sessler DI, Annadata R, Dechert M, Christensen R, Bjorksten AR. Midazolam minimally impairs thermoregulatory control. Anesth Analg 1995; 81: 393-398.

7. Mufti TM. Changes in body temperature during anaesthesia. J Pak Med Assoc 2002; 52: 220-224.

8. Ikeda T, Kazama T, Sessler DI, et al. Induction of anesthesia with ketamine reduces the magnitude of redistribution hypothermia. Anesth Analg 2001; 93: 934-938.

9. Isaacson DL, Yanosky DJ, Jones RA, Dennehy N, Spandorfer P, Baxter AL. Effect of MRI strength and propofol sedation on pediatric core temperature change. J Magn Reson Imaging 2011; 33: 950-956.

10. Tsui BC, Wagner A, Usher AG, Cave DA, Tang C. Combined propofol and remifentanil intravenous anesthesia for pediatric patients undergoing magnetic resonance imaging. Paediatr Anaesth 2005; 15: 397-401.

11. Kinoshita T, Suzuki M, Shimada Y, Ogawa R. Effect of low-dose ketamine on redistribution hypothermia during spinal anesthesia sedated by propofol. J Nippon Med Sch 2004; 71: 9298.

12. Thevaraja AK, Batra YK Rakesh SV, et al. Comparison of low-dose ketamine to midazolam for sedation during pediatric urodynamic study. Pediatr Anesth 2013; 23: 415-421.

13. Acar HV, Yarkan Uysal H, Gunal Eruyar S, Dikmen B. Body temperature decreases in pediatric patients undergoing magnetic resonance imaging under general anaesthesia. 10AP3-4. Eur J Anaesthesiol 2012: 29: 160 .
14. Lo C, Ormond G, McDougall R, Sheppards SJ, Davidson AJ. Effect of magnetic resonance imaging on core body temperature in anaesthetised children. Anaesth Intensive Care 2014; 42: 333-339.

15. Miller RD. Miller's Anesthesia (8th ed). Philadelphia: Saunders, 2015: 2763.

16. Patel A, Davidson M, Aziz R, Taylor T. Pediatric Temperature Variation in the MRI Scanner Under General Anesthesia (Abstract) PA/AAP Pediatric Anesthesiology 2011- San Diego CA: Winter Meeting, 2011.

17. Bryan YF, Templeton TW, Nick TG, Szafran M, Tung A. Brain magnetic resonance imaging increases core body temperature in sedated children. Anesth Analg 2011; 102: 1674-1679.

18. Kussman BD, Mulkern RV, Holzman RS. Iatrogenic hyperthermia during cardiac magnetic resonance imaging. Anesth Analg 2004; 99: 1053-1055.

19. Jenkins E, Hebbar KB, Karaga KK, et al. Experience with the use of propofol for radiologic imaging in infants younger than 6 months of age. Pediatr Radiol 2017; 47: 974- 983.

20. Leslie K, Sessler DI, Bjorksten AR, et al. Propofol causes a dose-dependent decrease in the thermoregulatory threshold for vasoconstriction but has little effect on sweating. Anesthesiology 1994; 81 : 353-360.

21. Matsukawa T, Kurz A, Sessler DI, Bjorksten AR, Merrifield B, Cheng C. Propofol linearly reduces the vasoconstriction and shivering thresholds. Anesthesiology 1995; 82: 1169-1180.

22. Diaz M, Becker DE. Thermoregulation: physiological and clinical considerations during sedation and general anesthesia. Anesth Prog 2010; 57: 25-33.

23. Miller RD. Miller's Anesthesia (8th ed). Philadelphia: Saunders, 2015: 1624.

24. Xiong J, Kurz A, Sessler DI, et al. Isoflurane produces marked and nonlinear decreases in the vasoconstriction and shivering thresholds. Anesthesiology 1996; 85: 240-245.

25. Sessler DI, McGuire J, Hynson J, Moayeri A, Heier T. Thermoregulatory vasoconstriction during isoflurane anesthesia minimally decreases cutaneous heat loss. Anesthesiology 1992; 76: 670-675.

26. KurzA, XiongJ, SesslerDI, DechertM, NoyesK, Belani $\mathrm{K}$. Desflurane reduces the gain of thermoregulatory arteriovenous shunt vasoconstriction in humans. Anesthesiology 1995; 83: 1212-1219. 
27. Kurz A, Plattner O, Sessler DI, Huemer G, Redl G, Lackner F. The threshold for thermoregulatory vasoconstriction during nitrous oxide/isoflurane anesthesia is lower in elderly than in young patients. Anesthesiology 1993; 79: 465-469.

28. Lenhardt R, Negishi C, Sessler DI, Ozaki M, Tayefeh F, Kurz A. Paralysis only slightly reduces the febrile response to interleukin-2 during isoflurane anesthesia. Anesthsiology 1998; 89: 648-656.

29. Lenhardt R, Negishi C, Sessler DI, et al. The effect of pyrogen administration on sweating and vasoconstriction thresholds during desflurane anesthesia. Anesthesiology 1999; 90: 1587-1595.

30. Negishi C, Lenhardt R, Sessler DI, et al. Desflurane reduces the febrile response to administration of interleukin-2. Anesthesiology 1998; 88: 1162-1169.

31. Ozaki M, Sessler DI, Suzuki H, Ozaki K, Atarashi K, Negishi C. The threshold for thermoregulatory vasoconstriction during nitrous oxide/sevoflurane anesthesia is lower in elderly than in young patients. Ann N Y Acad Sci 1997; 15: 789-791.
32. Saito T. A comparison of the body temperature during sevoflurane anesthesia and isoflurane anesthesia. Ann N Y Acad Sci 1997; 15: 786-788.

33. Sessler DI. Perioperative thermoregulation and heat balance. Lancet 2016; 387: 2655-2664.

34. Son SC, Yoon SH. Effects of propofol and ketamine on body temperature during induction of general anesthesia in children. A-618. Eur J Anaesthesiol 2004; $21: 151$.

35. López KR, Gibbs PH, Reed DS. A comparison of body temperature changes due to the administration of ketamine-acepromazine and tiletamine-zolazepam anesthetics in cynomolgus macaques. Contemp Top Lab Anim Sci 2002; 41: 47-50. 